MS23-02

\section{Electron tomography of radiation sensitive 3D nano-crystals in imaging and diffraction mode}

Jan Pieter Abrahams ${ }^{1}$, Max Clabbers ${ }^{2}$, Eric van Genderen ${ }^{3}$, Thorsten Blum $^{3}$

1. C-CINA, Basel University and LNB, Paul Scherrer Institute, Basel and Villigen, Switzerland

2. C-CINA, Basel University, Basel, Switzerland

3. LNB, Paul Scherrer Institute, Villigen, Switzerland

email: Jan-Pieter.Abrahams@psi.ch

Any electron microscope allows collecting electron diffraction data both in diffraction and in imaging mode. Both modes have their strengths and weaknesses. The advantage of electron imaging is that it provides spatial phase information of the sample, but at the expense of contrast. The advantage of electron diffraction is that it provides high contrast, but at the expense of spatial phase information. Here, we discuss how to exploit the strengths of both methods and how to combine them, in order to infer all the required information of radiation sensitive samples.

We show that by collecting in diffraction, we can measure up to 1000 times more useful data of weakly scattering, organic material, compared to imaging mode. We explain this significant difference from first principles and show that it is an intrisic limitation of imaging. Thus, we can explain why it is possible to collect a full three-dimensional high-resolution electron diffraction data set of a single protein nano-crystal, with the same number of electrons that is required for just a single high-resolution image of the same crystal.

Diffraction data lacks spatial phase information. Such information can be inferred from prior information of the sample, but when this is not available, it needs to be measered experimentally. One way of measuring spatial phases in three dimensions, is by electron tomography in imaging mode. Although wasteful in terms of electron dose (and hence causing increased radiation damage), it does provide independent phase information. We discuss recent results.

References:

[1] Clabbers, MTB \& Abrahams JP (2018) "Electron diffraction and three-dimensional crystallography for structural biology" (2018) Crystallography Reviews, DOI: 10.1080/0889311X.2018.1446427

Keywords: Electron diffraction, protein crystallography, electron tomography
MS23-03

\section{Low-dose scanning electron diffraction and pharmaceutical nanostructure}

Duncan Johnstone ${ }^{1}$, Christopher S. Allen ${ }^{2}$, Mohsen Danaie ${ }^{2}$, Royston C.B. Copley ${ }^{3}$, Anais Lafontaine ${ }^{4}$, Jeffrey Brum ${ }^{3}$, Angus I. Kirkland ${ }^{2}$, Paul A. Midgley ${ }^{1}$

1. Department of Materials Science and Metallurgy, University of Cambridge, Cambridge, United Kingdom

2. Electron Physical Sciences Imaging Centre (ePSIC), Diamond Light Source Ltd \& Department of Materials, University of Oxford, Oxford, United Kingdom

3. Physical Properties, Analytical Sciences and Development, GlaxoSmithKline Pharmaceuticals, Stevenage, United Kingdom

4. Evreux Materials Science, GlaxoSmithKline, Evreux, France

email: dnj23@cam.ac.uk

The active ingredients and excipients formulated together in pharmaceutical products are often organic crystals. Whilst $\mathrm{X}$-ray diffraction is ideal for probing the atomic structure of these organic crystals, it has limited use in investigating any heterogeneity of the pharmaceutical at the nanoscale [1]. Such 'nanostructure' may affect product performance and can, in principle, be probed by transmission electron microscopy but, until recently, beam damage has proven to be a major limiting factor [1]. Scanning electron diffraction (SED) is a new approach to acquiring nanoscale data from beam-sensitive materials [2,3]. SED involves the acquisition of a two-dimensional electron diffraction pattern at every probe position as a nm-sized electron probe is scanned across the specimen. If each pattern can be acquired before significant structural degradation (typically a few $\mathrm{ms}$ ), the 4D-SED dataset contains a wealth of nanoscale crystallographic data, making the technique a highly versatile hybrid diffraction-imaging approach.

Here, we report on the development of low-dose SED, in which diffraction patterns from pharmaceutical organic solids were recorded with an electron fluence of $\sim 5 \mathrm{e} / \AA^{2}$ and a spatial resolution of $\sim 5 \mathrm{~nm}$. Such low dose acquisition is greatly enhanced by the use of direct electron detectors and represents a reduction of approximately 2 orders of magnitude compared to previous SED of soft materials $[2,3]$. In particular, we used the Merlin-medipix detector system, a counting type detector, particularly suited to this kind of diffraction pattern acquisition owing to essentially zero noise acquisition and high dynamic range. A probe current of $1.6 \mathrm{pA}$ and a dwell time of $1 \mathrm{~ms}$ per probe position was used in the SED acquisition. A highly parallel $(\sim 0.6 \mathrm{mrad}$ convergence angle) beam was used and a reciprocal space structural resolution of better than $1 \AA^{-1}$ was obtained.

Results will be presented from a number of off the shelf active pharmaceutical ingredients, namely bicalutamide, acetaminophen, and probucol, as well as excipients lactose monohydrate and magnesium stearate. These materials span a range of reported characteristic doses and SED data were successfully obtained in all cases. For the most beam sensitive materials, the acquisition protocol is such that repeated scanning of the same area reveals almost complete destruction of the crystallinity - i.e. a 'diffract and destroy' approach. Nevertheless, high-quality diffraction data are obtained. Therefore, low-dose SED, as developed in this work, provides an 
essential platform for the investigation of nanostructure in pharmaceutical materials and other organic solids.

References:

[1] Eddleston, M. D. \& Jones, W., (2016), ch. 4, 103-134, WileyVCH Verlag GmbH \& Co.

[2] Johnstone, D. N. \& Midgley, P. A. (2017) Microsc. Microanal., 23, 1192-1193.

[3] Panova, O. et al., (2016) Micron, 88, 30-36.

Keywords: scanning electron diffraction, pharmaceuticals, nanostructure

\section{MS23-04}

\section{Possibility of improved phasing method on} Micro-ED

Tsumoru Shintake ${ }^{1}$, Cathal Cassidy ${ }^{1}$

1. OIST: Okinawa Institute Science and Technology, Graduate University, 1919-1 Tancha, Onna, Okinawa 904-0495, Japan email: shintake@oist.jp

The author discuss on basic physics of Bragg diffractions, and introduce a new way of image capturing on Bragg diffractions instead of Fraunhofer diffractions in a transmission electron microscope. There is a possibility to improve the phasing process on the protein crystallography (Micro-ED). In this scheme, we do not require standard phasing techniques; such as Multi-wavelength Anomalous Diffraction (MAD) method used in X-ray crystallography. The author also discuss on limitations on this scheme.

Keywords: Micro-ED, Phasing 